typeset using JPSJ.sty $<$ ver.0.8 $>$

\title{
Luttinger-liquid Parameter of Hubbard Chain and Hubbard Ladder
}

\author{
Kazuhiro SANO \\ Department of Physics Engineering, Mie University, Tsu, Mie 514-8507, Japan
}

(Received )

\begin{abstract}
We study the Luttinger-liquid parameter $K_{\rho}$ of the Hubbard chain and the Hubbard ladder models by the ordinary perturbation method combined with the Luttinger-liquid relation. According to the Luttinger-liquid relation, the critical exponent $K_{\rho}$ is related to the charge susceptibility $\chi_{c}$ and the Drude weight $D$ by $K_{\rho}=\frac{1}{2}\left(\pi \chi_{c} D\right)^{1 / 2}$. By calculating these quantities with the perturbation method, we obtain $K_{\rho}$ at the first-order analytically and up to the second-order numerically. We compare these results with results of the Bethe ansatz for the Hubbard chain and that of the numerical diagonalization for the Hubbard ladder. It shows that the perturbation calculation of $K_{\rho}$ is reliable in the weak coupling regime.
\end{abstract}

KEYWORDS: Luttinger-liquid, perturbation, Hubbard Ladder,superconductivity

\section{§1. Introduction}

Low-dimensional strongly correlated electron systems have been investigated extensively due to the possible relevance to high- $T_{c}$ superconductivity. In particular, One-dimensional(1D) Hubbard-like models provide an important ground for understanding electron-correlation effects. It is well known that a single Hubbard chain has generic properties of Luttinger liquid state with gapless spin and charge modes for repulsive interaction. For attractive interaction, a superconducting state characterized by dominant paring correlations with a spin gap is realized. 4 , 2, 3 ,

Recently, weak coupling theory has been applied to the problem of ladder models, which are interesting as a first step towards $2 \mathrm{D}$ systems and may be relevant for some materials. The theory reveals that the systems remain typical non-Fermi-liquid properties as $1 \mathrm{D}$ electronic systems 4,5 , 1) At half-filling, they are Mott insulators, exhibiting gaps to all excitations. Upon doping, the gaps survive except one gapless charge mode and a superconducting ( $\mathrm{SC}$ ) paring correlation characterized slowly power-low decay appears. According to the Luttinger-liquid theory, critical exponents of various types of correlation functions are determined by a single parameter $K_{\rho} .1$, 2.3) It is predicted that the SC correlation function of ladder systems is dominant for $K_{\rho}>0.5$ at lightly doping. It decays as $\sim r^{-\left(\frac{1}{2 K_{\rho}}\right)}$, whereas the " $4 k_{F}$ " charge density wave (CDW) correlation function decays as $\sim r^{-2 K_{\rho}}$. The spin density wave (SDW) and "2 $k_{F}$ " CDW correlation functions decay exponentially. 45 , 6

In spite of the good understanding of the single Hubbard chain which is exactly solved by the Bethe ansatz method, Hubbard ladder models are much less known. In fact, systematic treatment of $K_{\rho}$ as a function of interaction $U$ is not yet obtained. In this work we propose a simple method for calculating the Luttinger-liquid parameter $K_{\rho}$. It is the first and second-order perturbation expansion with respect to $U$ combined with the
Luttinger-liquid relation. These results are compared with the Bethe ansatz and the numerical diagonalization results.

\section{§2. Perturbation expansion of $K_{\rho}$}

In the Luttinger liquid theory, some relations have been established as universal relations in onedimensional models 1 , 2, 3) The critical exponent $K_{\rho}$ is related to the charge susceptibility $\chi$ and the Drude weight $D$ by

$$
K_{\rho}=\frac{1}{2}(\pi \chi D)^{1 / 2}
$$

with

$$
\chi^{-1}=\frac{1}{N} \frac{\partial^{2} E_{g}(n)}{\partial n^{2}}, \quad D=\frac{\pi}{N} \frac{\partial^{2} E_{g}(\phi)}{\partial \phi^{2}},
$$

where $N$ is the number of lattice site, $n$ is electron density and $E_{g}$ is the total energy of the ground state as a function of $n$ and a magnetic flux $\phi$. 3) If we calculate the ground state energy in some way, we can obtain $K_{\rho}$ through $\chi$ and $D$.

We apply the ordinary perturbation expansion in powers of $U$ to $E_{g}$,

$$
E_{g}=E_{0}+e_{1}+e_{2}+\ldots \ldots,
$$

where $E_{0}$ is the ground state energy of the noninteracting system and $e_{i}(i=1,2, \ldots)$ is the $i$-th order correction of $E_{g}$. According to the perturbation expansion of $E_{g}, \chi^{-1}$ and $D$ are given by

$\chi^{-1}=\chi_{0}^{-1}+x_{1} U+x_{2} U^{2} \ldots, \quad D=D_{0}+d_{1} U+d_{2} U^{2}+\ldots$. where $\chi_{0}^{-1}$ and $D_{0}\left(=4 \chi_{0}^{-1} / \pi\right)$ are the inverse charge susceptibility and the Drude weight of the non-interacting system respectively. Coefficients $x_{i}$ and $d_{i}(i=1,2, \ldots)$ are the $i$-th order corrections of $\chi^{-1}$ and $D$, which are determined by the second differential coefficients of $e_{i}$ with respect to $n$ and $\phi$ respectively. In the Hubbard model, the first-order term $e_{1}$ is easily obtained as $e_{1}=U \sum_{i}<n_{i \uparrow}><n_{i \downarrow}>=U N n^{2} / 4$. It leads 
$x_{1}=1 / 2$ and $d_{1}=0$, where $e_{1}$ is independent of the flux $\phi$.

Substituting these values into eq. (1), we obtain $K_{\rho}$ within the first perturbation expansion:

$$
1 / K_{\rho}^{2} \simeq 1+\frac{\chi_{0}}{2} U
$$

It shows that $K_{\rho}$ generally decreases with increasing the repulsive interaction $U$ in the weak coupling limit. It is noted that this approximation is equivalent to the Hartree-Fock (HF) approximation. The second-order term of the ground state energy $e_{2}$ is also easily obtained as

$e_{2}=\frac{U^{2}}{N} \sum_{k_{1}, k_{2}, q, \sigma} \frac{f\left(k_{1}\right) f\left(k_{2}\right)\left(1-f\left(k_{1}+q\right)\right)\left(1-f\left(k_{2}-q\right)\right)}{\varepsilon\left(k_{1}\right)+\varepsilon\left(k_{2}\right)-\varepsilon\left(k_{1}+q\right)+\varepsilon\left(k_{2}-q\right)}$ where $\varepsilon(k)$ is the non-interacting band and $f(k)=$ $\theta\left(k_{F}-|k|\right)$. Using $e_{2}$, we can calculate $K_{\rho}$ up to order $U^{2}$ by

$$
1 / K_{\rho}^{2} \simeq 1+\frac{\chi_{0}}{2} U+\left(x_{2}-\frac{\pi}{4} d_{2}\right) \chi_{0} U^{2},
$$

with

$$
x_{2}=\frac{1}{N U^{2}} \frac{\partial^{2} e_{2}}{\partial n^{2}}, \quad d_{2}=\frac{\pi}{N U^{2}} \frac{\partial^{2} e_{2}}{\partial \phi^{2}} .
$$

In the following section, we will estimate the above integral and obtain $x_{2}$ and $d_{2}$ numerically.

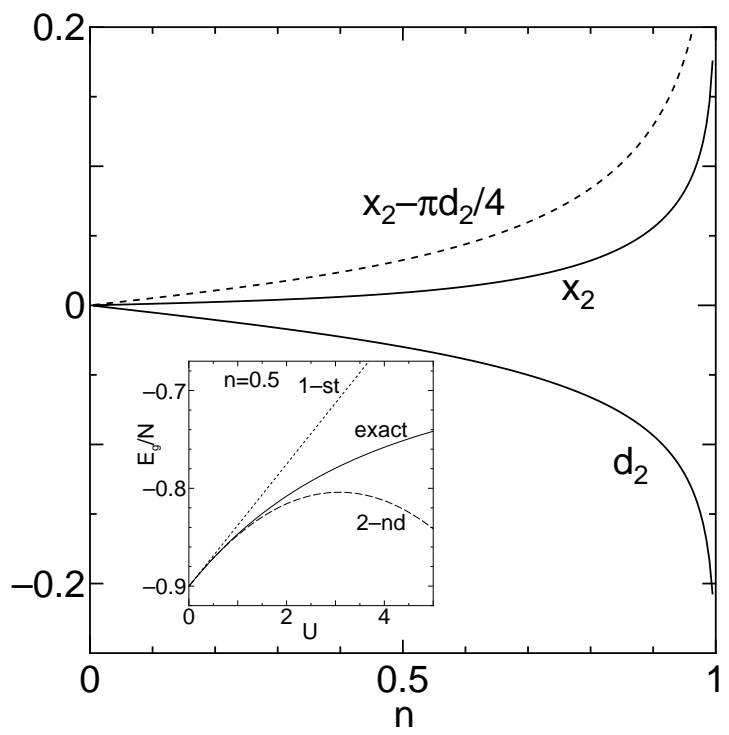

Fig. 1. The coefficients of the second-order terms $x_{2}$ and $d_{2}$ as a function of $n$. The broken line represents $\left(x_{2}-\frac{\pi}{4} d_{2}\right)$. The inset shows the ground state energy of the system as a function of $U$ by the first-order and the second-order perturbation calculation with the exact result at quarter-filling.

\section{§3. Hubbard chain and Hubbard ladder}

At first, we examine the Hubbard chain model whose non-interacting band is given by $\varepsilon(k)=-2 t \cos k$. In this case, we have $\chi_{0}^{-1}=\pi t \sin k_{F}$ and $K_{\rho}$ up to the first-order expansion of $U$

$$
1 / K_{\rho}^{2}=1+U / 2 \pi t \sin k_{F},
$$

where $k_{F}=\frac{\pi}{2} n$ is the Fermi wave number. This expression of $K_{\rho}$ is exactly equal to the result of bosonization method for the Hubbard chain.

To estimate $e_{2}$, we use $N=200$ and 400 sites systems. We confirm that the size dependence of $x_{2}$ and $d_{2}$ are very small and negligible. In Fig.1, we show $x_{2}$ and $d_{2}$ as a function of $n$. These values seem to diverge at the limit $n \rightarrow 1$. It might reflect the insulator transition of the Hubbard chain at half-filling. We also show the ground state energy $E_{g} / N$ as a function of $U$ at quarterfilling. The result of $E_{g} / N$ is consistent with the exact result in the weak coupling region. In Figs.2(a) and (b),
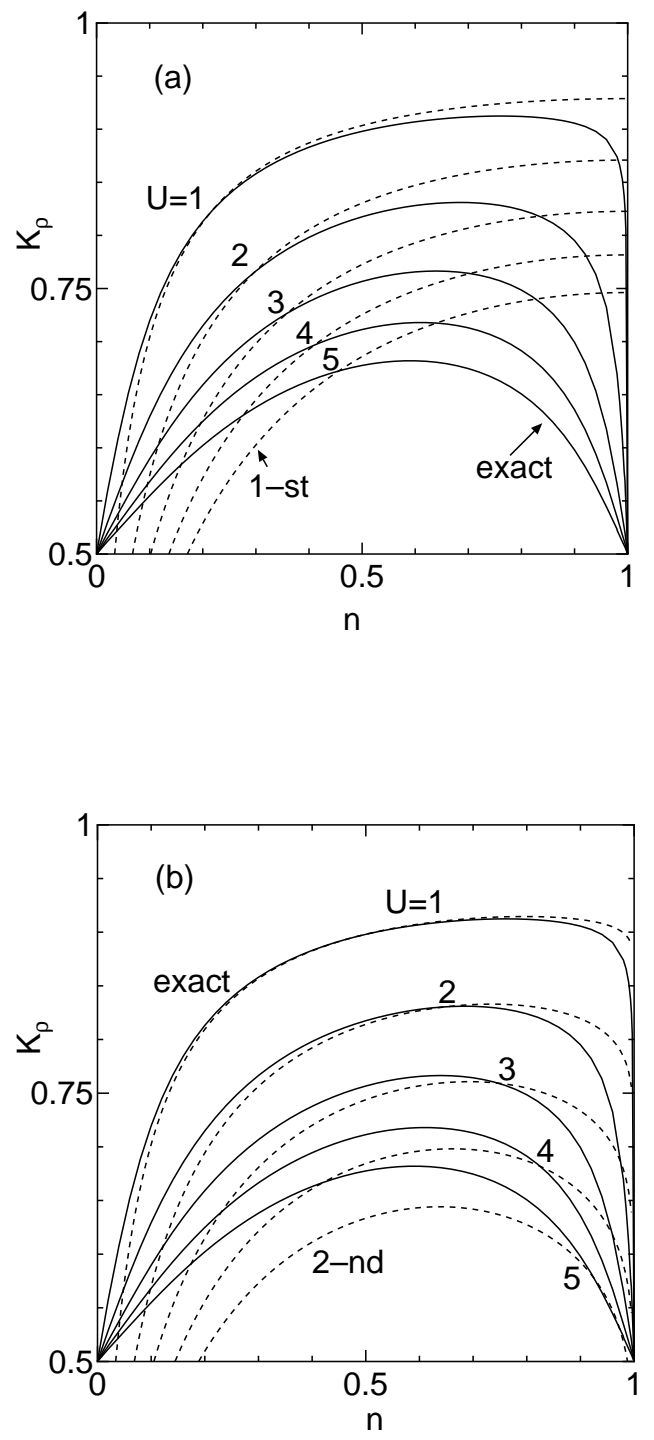

Fig. 2. $\quad K_{\rho}$ as a function of $n$ by (a) the first-order and (b) the second-order perturbation expansion (b) with the exact result of the Bethe ansatz. The solid lines represent the results of the Bethe ansatz and the broken lines are that of the perturbation expansion.

we show $K_{\rho}$ by the perturbation expansion with the ex- 
act result of the Bethe ansatz.10.11 They show that the result of the perturbation expansion is consistent with the exact result in the weak coupling region. It also indicates that the second-order perturbation calculation agrees with the exact solution more than the first-order calculation in the weak coupling regime. In the strong coupling regime, $K_{\rho}$ of the first-order calculation seems to be close that of the exact solution at $n \sim 0.4$. However, it may be an accidental.

Next, we consider the Hubbard ladder Hamiltonian

$$
\begin{aligned}
H & =-t_{l} \sum_{i, \alpha, \sigma} c_{i, \alpha, \sigma}^{\dagger} c_{i+1, \alpha \sigma}-t_{r} \sum_{i, \sigma} c_{i, 1, \sigma}^{\dagger} c_{i, 2, \sigma}+h . c . \\
& +U \sum_{i, \alpha} n_{i, \alpha \uparrow} n_{i, \alpha \downarrow},
\end{aligned}
$$

where $c_{i, \alpha, \sigma}^{\dagger}$ stands for a creation operator of an electron with spin $\sigma$ at site $(i, \alpha)$ and $U$ is the on-site interaction. Here, $\alpha(=1,2)$ denotes legs and $i$ is rung. In this case, non-interacting band is written as

$$
\varepsilon^{ \pm}(k)=-2 t_{l} \cos k \pm t_{r},
$$

where $\varepsilon^{+}(k)\left(\varepsilon^{-}(k)\right)$ represents the upper (lower) band and $k$ is the wave vector. If we define $k_{F-}\left(k_{F+}\right)$ as a Fermi point in the lower (upper) band, we find that $\varepsilon^{-}\left(k_{F-}\right)=\varepsilon^{+}\left(k_{F+}\right)$ with $k_{F-}+k_{F+}=k_{F}=n \pi$. The differential coefficient of $\varepsilon^{-}\left(k_{F-}\right)$ with respect to $n$ leads the inverse charge susceptibility $\chi_{0}^{-1}$. After a bit of calculation, we get

$$
\begin{aligned}
\chi_{0}^{-1} & =-t_{l} \pi \sin k_{F}\left\{\frac{\cos k_{F}\left(1-\cos k_{F}\right)+\left(t_{r} / t_{l}\right)^{2}-\sin ^{2} k_{F}}{\left(1-\cos k_{F}\right)^{2}}\right\} \\
& \times\left\{\left(t_{r} / t_{l}\right)^{2}-2 \frac{\left(t_{r} / t_{l}\right)^{2}-\sin ^{2} k_{F}}{1-\cos k_{F}}\right\}^{-1 / 2} .
\end{aligned}
$$

Substituting $\chi_{0}^{-1}$ to eq.(3), we have $K_{\rho}$ of the Hubbard ladder model analytically within the first-order perturbation method.22

When $n$ is smaller than $n_{c}$ which is determined by $\varepsilon^{+}(0)=\varepsilon^{-}\left(\frac{\pi n_{c}}{2}\right)$, electrons are filled only in the lower band. The density of state of the lower band is a half of that of the chain model. Then, $\chi_{0}$ is given by $1 /\left(2 \pi \sin k_{F}\right)$ which is a half of that of the chain model. Within the first-order perturbation calculation, the upper band is irrelevant and the correction of $K_{\rho}$ becomes a half of that of the chain model.

As well as the Hubbard chain model, we estimate the second-order terms $e_{2}, x_{2}$ and $d_{2}$ numerically.13) In Fig.3, we show $x_{2}, d_{2}$ and $x_{2}-\frac{\pi}{4} d_{2}$ as a function of $n$ at $t_{r} / t_{l}=1.0$. For $n>n_{c}=0.5$, the values of $x_{2}$ seem to be small, but the absolute values of $d_{2}$ are large. It shows that the values $x_{2}-\frac{\pi}{4} d_{2}$ are positive and large. It indicates that the second-order term of the repulsion $U$ reduces $K_{\rho}$ as well as the first-order term. This result does not contradict the behavior of the SC correlation function obtained by density matrix renormalization group and quantum Monte Carlo methods $14,15,16)$ Although system sizes used in these numerical works are too small to determine $K_{\rho}$ precisely, we find that a rough estimate indicates $0.5 \lesssim K_{\rho} \lesssim 1.0$.
On the other hand, for $n<0.5$, the values $x_{2}-\frac{\pi}{4} d_{2}$ are negative. In particular, the absolute values are very large near $n=0.5$. It indicates that $K_{\rho}$ is enhanced by the second-order term. In contrast to the case for $n>0.5$, the existence of the upper band seems to produce an effective attraction in the second-order correction. This result may consist with an enhancement of the SC correlation shown by the numerical diagonalization method.17) Figure 4 indicates $K_{\rho}$ as a function of $n$ at $t_{l} / t_{r}=1.0$ by the first- and the second-order perturbation calculation.

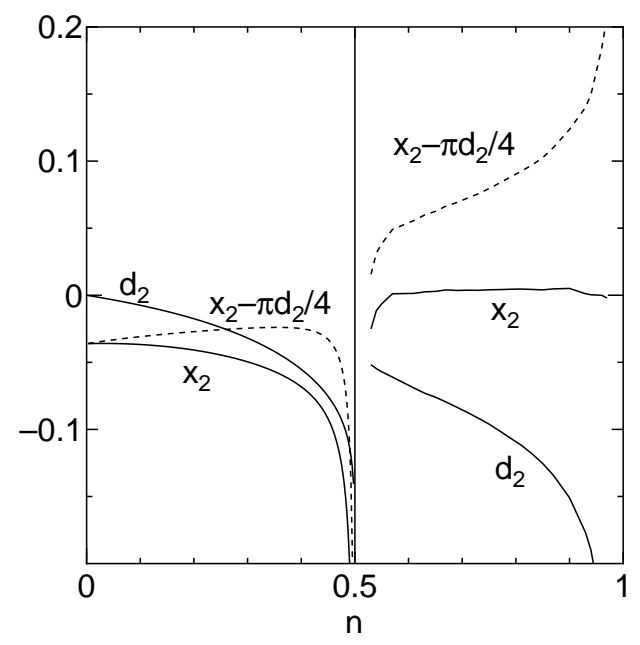

Fig. 3. The coefficients $x_{2}$ and $d_{2}$ as a function of $n$ for the Hubbard ladder model. The broken lines represent $\left(x_{2}-\frac{\pi}{4} d_{2}\right)$.

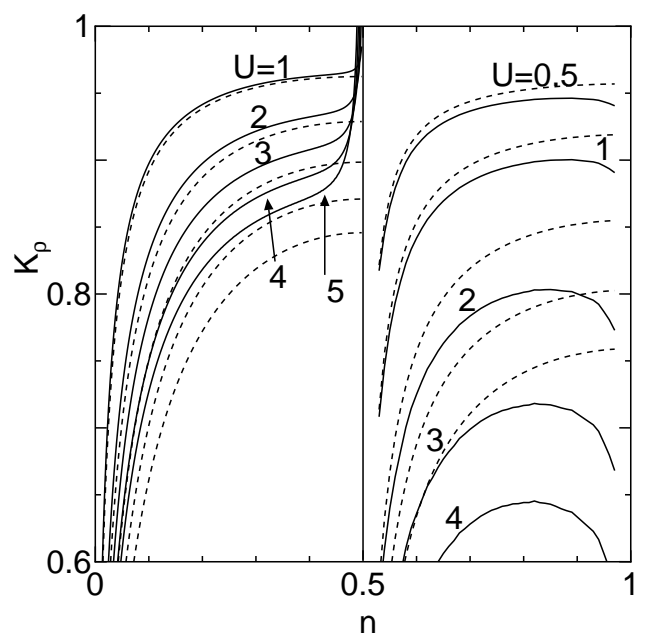

Fig. 4. $\quad K_{\rho}$ as a function of $n$ for the Hubbard ladder model by the first-order (the broken lines) and the second-order (the solid lines) perturbation expansion.

To clarify the validity of the perturbation expansion of $K_{\rho}$, we examine a finite size system of the ladder model. We numerically diagonalize the Hamiltonian of 14 sites (7 unit cells) system by using the Lanczos algorithm. 
We use the periodic boundary condition for $N_{e}=8$, the Moebius boundary condition for $N_{e}=10$ and the antiperiodic boundary condition for $N_{e}=12$, where $N_{e}$ is the total electron number.18 This choice of the boundary condition gives either fully occupied or empty single particle orbitals ( closed shell ) and removes accidental degeneracy in the non-interacting case. The uniform charge susceptibility $\chi_{c}$ and the Drude weight $D$ is calculated from the ground state energy with the usual method.

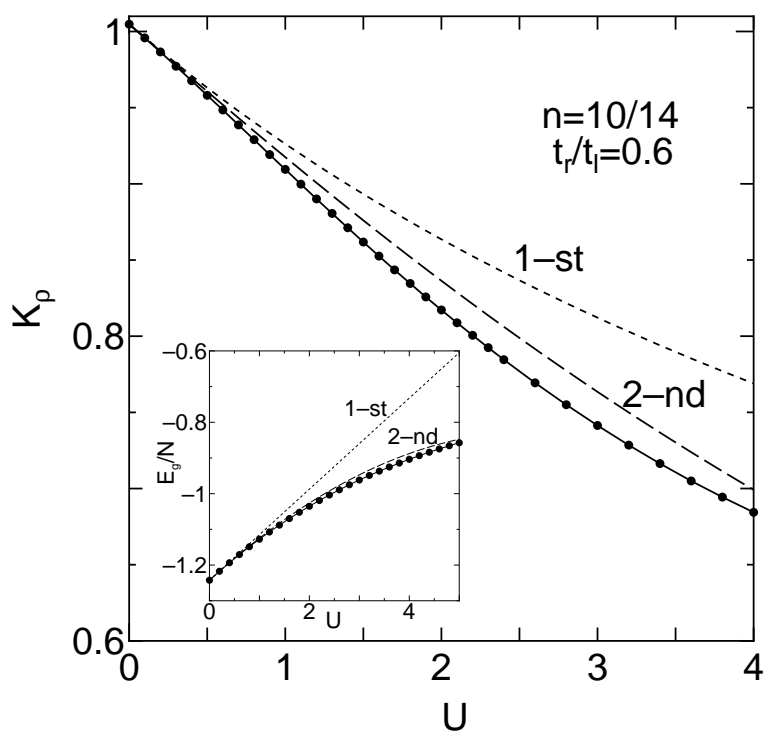

Fig. 5. $K_{\rho}$ of the finite size system for the Hubbard ladder as a function of $U$. The solid circles represent the result of the numerical diagonalization for 7 rungs system at $t_{r} / t_{l}=0.6$. The broken line represents the result of the first-order perturbation expansion and the dashed line is that of the second-order perturbation expansion. The inset shows the ground state energy of the system as a function of $U$ by the first-order and the secondorder perturbation calculations with the result of the numerical diagonalization.

In Fig.5, we show $K_{\rho}$ and the ground state energy $E_{g} / N$ of the finite size system with the result of the numerical diagonalization method. In the weak coupling regime, the results of the perturbation approximation and the numerical diagonalization are in agreement with each other. It also shows that the second-order perturbation is better than the first-order in the weak coupling regime.

\section{$\S 4$. Summary and discussion}

In this work we examine the Luttinger-liquid parameter $K_{\rho}$ of the Hubbard chain and the Hubbard ladder models by the ordinary perturbation method combined with the Luttinger-liquid relation. According to the Luttinger-liquid relation, we obtain $K_{\rho}$ at the firstorder analytically and up to the second-order numerically. Comparing $K_{\rho}$ with the exact result of Bethe ansatz and that of the numerical diagonalization method, we show that the analysis of perturbation method is reliable in the weak coupling region.

Generally speaking, the validity of the perturbation expansion is not always obvious in 1D electron systems. 19) However, it has been analytically shown that the ordinary perturbation expansion of the ground state energy of the Hubbard chain agrees with the expansion exact Bethe ansatz solution at half-filling.20 21, 22 Although the convergence radius is zero, the perturbation is possible as an asymptotic expansion.

Away from half-filling, the integral equations of the Bethe ansatz solution are not analytically solved. However, we confirm that $e_{2}$ consists with the second-order term of $U$ in the Bethe ansatz solution numerically. It suggests that the perturbation expansion in powers of $U$ is also possible as an asymptotic expansion.23

[1] J. Solyom: Adv. Phys. 28 (1979) 209.

[2] F.D.M. Haldane: J. Phys. C14 (1981) 2585.

[3] J. Voit: Rep. Prog. Phys. 58 (1995) 977.

[4] M. Fabrizio: Phys. Rev. B 48 (1993) 15838.

[5] H. J. Schulz: Phys. Rev. B 53 (1996) R2959.

[6] L. Balentz and M. P. A. Fisher: Phys. Rev. B 53 (1996) 12133.

[7] K. Sano and Y. Ōno: J. Phys. Soc. Jpn. 67 (1998) 4151.

[8] K. Sano: Proc. LI-st YAMADA conference on SCES, Nagano, 1999, Physica B (to be published).

[9] From a study of the conductance in the 1D interacting electron system, similar result is obtained; A. Kawabata: J. Phys. Soc. Jpn. 65 (1996) 30.

[10] E.H. Lieb and F.Y. Wu: Phys. Rev. Lett. 20 (1968) 1443.

[11] H. J. Schulz: Phys. Rev. Lett. 64 (1990) 2831.

[12] It is noted that application of our analysis is limited to systems which have one gapless charge mode at the low energy limit. The number of the gapless charge mode is not always one in the ladder model. See ref. [4].

[13] For $n>n_{c}$, finite size effect of $x_{2}$ is large because Fermi points exist in both of the upper and the lower bands. To reduce it, we fit $e_{2}$ to a polynomial of $n$ with various density points and estimate $x_{2}$ by the second-order coefficient of the polynomial.

[14] R. M. Noack and S. R. White: Phys. Rev. Lett. 73 (1994) 882.

[15] K. Kuroki, T. Kimura and H. Aoki: Phys. Rev. B 54 (1996) R15641.

[16] S. Daul, D. J. Scalapino and S. R. White: cond-mat/9911290.

[17] K. Yamaji, Y. Shimoi and T. Yanagisawa: Physica C235-240 (1994) 2221; They claimed that the superconducting state appears in the region where the lowest unoccupied level of the upper band is close to the highest occupied level of the lower band.

[18] Detail of the Moebius boundary condition is discussed by T.F.A. Muller and T. M. Rice: Phys. Rev. B 58 (1998) 3425.

[19] M. Ogata and H. Shiba: Phys. Rev. B 41 (1990) 2326. They discussed the momentum distribution function $n(k)$ and spin correlation function $S(k)$ of the Hubbard chain model by the perturbation calculation. They showed that these functions have not a power-law singularity but a logarithmic singularity.

[20] M. Takahasi: Prog. Theor. Phys. 45 (1971) 2137.

[21] E. N. Economou and P.N. Poulopulous: Phys. Rev. B 20 (1979) 4756.

[22] W. Metzner and D. Vollhardt: Phys. Rev. B 39 (1989) 4462.

[23] If the convergence radius of the perturbation expansion is not zero, the electronic state of the system should be not "Luttinger-liquid" but "Fermi-liquid" in the weak coupling limit. 